\title{
Differentiation of human CD14+ monocytes: an experimental investigation of the optimal culture medium and evidence of a lack of differentiation along the endothelial line
}

\author{
Wajima Safi ${ }^{1}$, Andreas Kuehnl ${ }^{1}$, Andreas Nüssler ${ }^{2}$, Hans-Henning Eckstein ${ }^{1}$ and Jaroslav Pelisek ${ }^{1}$
}

\begin{abstract}
The aim of this study was to determine the optimal culturing media for human CD14+ monocytes and to evaluate whether these cells are capable of differentiating into vascular endothelial cells. Human monocytes isolated from peripheral blood were cultured for $1,3,7,10$ or 14 days in different media containing either $10 \%$ fetal bovine serum (FBS), $10 \%$ autologous donor serum (Auto), 10\% FBS with interleukin-3 and macrophage colony stimulating factor (FBS-WF) or 10\% Auto and the same growth factors (AU-WF). The cells were differentiated using endothelial cell conditioning medium (EC). Viability was measured using the MTT (3-(4,5-dimethylthiazol-2-yl)-2,5-diphenyltetrazolium bromide) assay, and the cells were characterized by histology, immunohistochemistry and western blot analysis. Monocytes treated with Auto, FBS-WF or AU-WF medium generated a significant higher yield of vital cells after 7 days in culture compared with FBS-only medium (mean difference (MD) $=0.318$, $P=0.01 ; \mathrm{MD}=1.83, P=0.04$; or $\mathrm{MD}=0.271, P=0.01$ and $\mathrm{MD}=0.318, P=0.102)$. All tested media led to the differentiation of monocytes into macrophages, identified by CD68, especially in the FBS-WF medium $(\mathrm{MD}=+18.3 \% ; P=0.04$ ). Differentiation into ECs caused a significant decrease in cell viability in all media. Endothelial cell markers, including CD31, CD144, VEGF, VEGF-R2 and CD34, could not be detected. Autologous serum significantly increases the yield of monocytederived cells with a higher effectiveness than commonly used FBS-only serum. There is no further benefit in culturing monocytes longer than 7 days. The cultivation of monocytes in the tested media leads preferentially to differentiation into macrophages. Differentiation into endothelial cells did not take place.
\end{abstract}

Experimental \& Molecular Medicine (2016) 48, e227; doi:10.1038/emm.2016.11; published online 15 April 2016

\section{INTRODUCTION}

Circulating monocytes are not yet fully differentiated cells and have the potential to transform into various other cell types such as macrophages, dendritic cells, liver Kupffer cells or even microglia in the central nervous system. ${ }^{1-4}$ For a long time, it was believed that monocytes could only differentiate into cell types possessing a phagocytic capacity. However, several recent studies indicate that CD14+ monocytes have the ability to express lineage markers other than their own when treated with specific additives. Under stimulation with M-CSF and RANKL, they can, for example, differentiate into osteoclasts. ${ }^{5-7}$ Kuwana et al. ${ }^{8-10}$ reported that monocytes are capable of transdifferentiating into a progenitor cell population that is then able to differentiate into several mesenchymal cell types, including bone, fat, neurons and so on.
Endothelial lineage markers such as CD144, vascular endothelial growth factor (VEGF) and VEGF receptor 2 (VEGF-R2) are co-expressed in vitro by CD14+ monocytes in response to incubation with a combination of various angiogenic factors such as VEGF. ${ }^{5,6}$ Harraz et al. ${ }^{7}$ also reported that CD14+ monocytes also have the potential to be incorporated into the endothelium of blood vessels and to transdifferentiate into endothelial cells. Several other studies investigated the role of monocyte-derived circulating endothelial cell progenitors in neovasculogenesis in human beings. ${ }^{11,12}$ These cells, however, were $\mathrm{CD} 14+$ negative and constituted a phenotypically distinct population of circulating endothelial cells. According to Kuwana et al. ${ }^{13}$ CD14+ monocytes isolated from the peripheral blood of patients first transform into a primitive cell population with a fibroblast-like morphology that are able to express

\footnotetext{
${ }^{1}$ Department of Vascular and Endovascular Surgery, Klinikum rechts der Isar der Technischen Universität München, München, Germany and ${ }^{2}$ Siegfried Weller Institute for Trauma Research, BG Trauma Center, Eberhard Karls University Tübingen, Tübingen, Germany

Correspondence: Dr W Safi or Dr J Pelisek, Department of Vascular and Endovascular Surgery, Klinikum rechts der Isar der Technischen Universitaet Muenchen, Ismaninger Strasse 22, D-81675 Munich, Germany.

E-mail: wajimasafi@aol.com or jaroslav.pelisek@tum.de

Received 9 January 2015; revised 17 November 2015; accepted 29 November 2015
} 
embryonic markers such as CD34 and CD45. This cell population was termed monocyte-derived multipotential cells (MOMC). When cultivated in specific growth medium, these MOMC differentiated into the endothelial cell lineage with their typical morphology. ${ }^{13}$ These data suggest that cultivating monocytes in a media favoring endothelial differentiation may lead to cells of the endothelial phenotype. ${ }^{8,9,13}$

The aim of our study was therefore to verify the differentiation of cultured human primary monocytes into the endothelial cell lineage and to assess the optimal cell culture conditions for monocytes and their differentiation into endothelial cells. For this purpose, the effect of different cell culture media and additives as well as the expression level of specific markers, such as CD14, CD68, CD144, CD106, VEGF-R2 and VEGF, on these monocyte-derived cells was analyzed.

\section{MATERIALS AND METHODS}

\section{Isolation of monocytes and cell culture}

Peripheral blood was obtained from healthy adults, and peripheral blood mononuclear cells were isolated by density gradient centrifugation using Histopaque $\left(1.077 \mathrm{~g} \mathrm{ml}^{-1}\right.$; Sigma-Aldrich, Munich, Germany) as previously described. ${ }^{13,14}$ All cells were cultured in RPMI 1640 medium (Biochrom; Berlin, Germany) supplemented with $1 \%$ penicillin/streptomycin in 6-, 12- and 96-well plastic plates. One of the following additions was made to the cell culture medium: (1) $10 \%$ fetal bovine serum (FBS) (Biochrom), (2) 10\% autologous serum from the corresponding donor, (3) 10\% FBS with the growth factors M-CSF (5.0 $\mathrm{ng} \mathrm{ml}^{-1}$ ) (R\&D Systems, Wiesbaden, Germany) and IL-3 $\left(0.4 \mathrm{ng} \mathrm{ml}^{-1}\right.$ ) (R\&D Systems) or (4) 10\% autologous serum with the growth factors M-CSF $\left(5.0 \mathrm{ng} \mathrm{ml}^{-1}\right)$ and IL-3 $\left(0.4 \mathrm{ng} \mathrm{ml}^{-1}\right)$. These media were tested with uncoated plates and with plates coated with $10 \mu \mathrm{gl}^{-1}$ fibronectin (Sigma-Aldrich).

The cells were allowed to adhere overnight in the chosen medium. The non-adherent cells were then removed by careful aspiration, and the remaining heterogeneous cell population, enriched with monocytes, was cultured in the selected medium for $1,3,7,10$ or 14 days at $37^{\circ} \mathrm{C}$ and $5 \% \mathrm{CO}_{2}$. The chosen medium was replaced by fresh medium of the same type every 2 days.

Cells that were supposed to differentiate into endothelial cells were cultured for a maximum of 7 days in the selected medium. After 7 days, the chosen growth medium was removed and the cells were treated for another 7 days with a commercially available endothelial cell conditioning medium (EC) (Provitro, Berlin, Germany) supplemented with FCS $(0.02 \mathrm{ml})$, ECGS/H $(4 \mu \mathrm{l})$, human recombinant EGF $(0.10 \mathrm{ng})$, human rec. basic fibroblast growth factor $(1 \mathrm{ng})$, hydrocortisone $(1 \mu \mathrm{g})$, gentamicin $(50 \mu \mathrm{g})$ and amphotericin b $(50 \mathrm{ng})$. A medium exchange was performed every 2 days.

The cells were cultured in 6- or 12-well plates and collected by mechanical scraping at day $1,3,7,10$ or 14 (1 plate from the same batch on each day). The collected cells were characterized by immunohistochemistry and western blotting. Cells cultured in 96-well plates were used for examining cell viability using the MTT (3-(4,5-dimethylthiazol-2-yl)-2,5-diphenyltetrazolium bromide) assay. Four independent experiments in triplicate were performed each time.

\section{Measurement of cell viability (MTT)}

On day $1,3,7,10$ or 14 , the cells were treated with $10 \%$ MTT (working concentration $0.5 \mathrm{mg} \mathrm{ml}^{-1}$ ), which is reduced by living cells to a non-soluble purple formazan. ${ }^{15,16}$ For the viability assay, the experiments were performed using five or six wells of cultured cells for each treatment. Afterwards, the cells were incubated for $3 \mathrm{~h}$ at $37^{\circ} \mathrm{C}$, and the reaction was stopped by an appropriate stop solution (bidest. water, $0.01 \mathrm{M} \mathrm{HCl}, 10 \%$ sodium dodecyl sulfate). The optical density of the cell suspensions was measured at $590 \mathrm{~nm}$.

\section{Cell characterization by histology and immunohistochemistry}

After removal of the growth medium, the cells cultured on 12-well plates were collected by scraping the cell culture dishes and were resuspended in $5 \mathrm{ml}$ phosphate-buffered saline (PBS) (Biochrom). After $5 \mathrm{~min}$ of centrifugation at $500 \mathrm{~g}$ at room temperature, the supernatant was removed, and the cell pellet was resuspended in $1 \mathrm{ml}$ of PBS. One micoliter of the cell suspension was transferred onto a slide and fixed with acetone.

The slides were stained with various primary antibodies and visualized using an LSAB-HRP kit (Dako, Hamburg, Germany) according to the manufacturer's instructions. To investigate whether the cultured cells were monocytes, we stained the cells with anti-CD68 antibodies (R\&D Systems, dilution 1:10000) as well as anti-CD14 antibodies (R\&D, 1:200) on days 1-14. Furthermore, the following primary antibodies (R\&D) were used: CD144 (1:200), VEGF (1:100), VEGF-R2 (1:200), CD31 (1:200) and CD34 (1:15). All slides were counterstained with hematoxylin and eosin. The stained cells were dehydrated in a series of graded ethanol solutions and then covered with a cover slip. The number of positive cells was counted using light microscopy (Axio Observer A1; Zeiss, Jena, Germany) at $\times 4$ magnification. The ratio of antibody-positive to antibody-negative cells on the stained slides was counted on days 1, 3 and 7 .

The cells were also cultured on chamber slides after centrifugation using acetone fixation to generate a better macroscopic picture of their morphology and their manner of growth.

\section{Cell characterization by western blot analysis}

Cells cultured in Auto, FBS-WF or AU-WF were harvested using trypsin and lysed as previously described. ${ }^{17}$ Briefly, sample loading buffer and reducing agent (both from Bio-Rad, Hercules, CA, USA) were added to the lysates. A standard of $5 \mu \mathrm{g}$ of protein was used from every sample. The samples were heated $\left(5 \mathrm{~min}, 95^{\circ} \mathrm{C}\right)$ and loaded on 4 to $15 \%$ Tris-HCl Criterion gels (Bio-Rad), separated by SDS-PAGE, and transferred to nitrocellulose. The membranes were probed for CD14 (dilution 1:1000, all antibodies by R\&D Systems), CD68 (1:500), CD31 (1:500), CD144 (1:100), CD106 (1:1000), VEGF (1:500, Thermo Fisher Scientific, Schwerte, Germany) and the housekeeping protein GAPDH (1:5000, Abcam, Cambridge, UK). The blocking and development procedures were performed in accordance with the manufacturer's (Bio-Rad) protocol. The samples were tested for the chosen antibodies on day 1, 3, 7 and 14 with at least 7 days of prior culturing in endothelial cell medium. Day 1 was set as $100 \%$ in all experiments, and the values at day 3, 7 , 10 and 14 are related to day 1 and display the increase or decrease in cell viability in comparison to the starting day. The proteins were visualized by a chemiluminescent detection method (SuperSignal West Pico Chemiluminescent Substrate, Thermo Scientific, Waltham, MA, USA) using Kodak Scientific Imaging (New Haven, CT, USA) film. The developed film was digitalized, and protein quantification was performed using the free software ImageJ $1.44 \mathrm{p}$ (the National Institutes of Health, Bethesda, MD, USA). Following normalization to GAPDH, the average of the control tissue samples was set as $100 \%$, and the results were calculated in comparison. 


\section{Statistical evaluation}

All results are presented as mean values and mean differences (MDs) with their s.d. The data were normally distributed, and therefore a Student's $t$-test was used for comparison. Significant differences among more than two groups were analyzed by one-way analysis of covariance. All statistical comparisons were two-sided with $P<0.05\left(^{*}\right)$ as the level of significance. In the Figures, the results are displayed as boxes-and-whisker plots. The middle line inside each box represents the median, the upper end of the box represents the 75th quartile, the lower end of the box represents the 25th quartile, the upper whisker represents the 97.5th quartile, the lower whisker represents the 2.5th quartile and open circles represent outliers beyond the 2.5th or 97.5th quartile. All statistical analyses were performed using SPSS for Windows version 17.0 software (SPSS, Chicago, IL, USA).

\section{RESULTS}

\section{Comparison of different cell culture conditions}

The cell viability of the cultured human monocytes was measured for up to 14 days to evaluate the optimal culture conditions. No significant differences were observed when cells were cultured with or without fibronectin-coated plates (data not shown); therefore, all cell culture experiments were performed without coating.

The cultured monocytes showed a significant increase in cell viability between day 1 and 7 in Auto $(\mathrm{MD}=0.318 ; P=0.01)$, AU-WF $(\mathrm{MD}=0.271 ; P=0.01)$ and FBS-WF $(\mathrm{MD}=0.243$; $P=0.01$ ) (Table 1). FBS-only medium did not generate a significant increase in viability $(\mathrm{MD}=0.104 ; P=0.102)$

Table 1 Cell viability values for each medium at OD $590 \mathrm{~nm}$ from day 1 to 7

Estimates

\begin{tabular}{llllll} 
& & & & & $95 \%$ confidence interval \\
\cline { 3 - 6 } Medium & & & & $\begin{array}{c}\text { Lower } \\
\text { bound }\end{array}$ & $\begin{array}{c}\text { Upper } \\
\text { bound }\end{array}$ \\
\hline Dependent variable: Abs & & & & & \\
OD_Auto & 1 & $0.259^{\mathrm{a}}$ & 0.034 & 0.192 & 0.326 \\
& 3 & $0.411^{\mathrm{a}}$ & 0.034 & 0.344 & 0.478 \\
OD_AU_WF & 7 & $0.577^{\mathrm{a}}$ & 0.034 & 0.510 & 0.644 \\
& 1 & $0.308^{\mathrm{b}}$ & 0.027 & 0.254 & 0.361 \\
OD_FBS & 3 & $0.441^{\mathrm{b}}$ & 0.027 & 0.387 & 0.495 \\
& 7 & $0.579^{\mathrm{b}}$ & 0.027 & 0.525 & 0.633 \\
OD_FBS_WF & 1 & $0.180^{\mathrm{c}}$ & 0.016 & 0.149 & 0.211 \\
& 3 & $0.248^{\mathrm{c}}$ & 0.016 & 0.217 & 0.279 \\
& 7 & $0.284^{\mathrm{c}}$ & 0.016 & 0.253 & 0.315 \\
& 1 & $0.215^{\mathrm{d}}$ & 0.027 & 0.161 & 0.268 \\
& 3 & $0.308^{\mathrm{d}}$ & 0.027 & 0.254 & 0.362 \\
& 7 & $0.458^{\mathrm{d}}$ & 0.027 & 0.404 & 0.511 \\
\hline
\end{tabular}

Abbreviation: OD, optical density.

${ }^{a}$ Covariates appearing in the model are evaluated at the following values: baseline $=0.2586$

${ }^{\mathrm{b}}$ Covariates appearing in the model are evaluated at the following values: baseline $=0.3076$

cCovariates appearing in the model are evaluated at the following values: baseline $=0.1800$.

dCovariates appearing in the model are evaluated at the following values: baseline $=0.2147$
(Figure 1a and Table 1). In the pair-wise comparisons, Auto $(P=0.02)$ generated a significantly higher yield of cells than FBS. Medium supplemented with autologous serum and growth factors showed the highest viability in absolute cell numbers at day 7 (Figure 1a). Interestingly, medium with autologous serum alone showed similar cellular viability as that supplemented with growth factors, as seen in Figure 1a. Further incubation in any selected medium until day 14 did not lead to any additional increase in cell viability (Figure $1 \mathrm{~b}$ ).

Based on the results above, cultured monocytes were treated with EC starting at day 7 for an additional 7-day period. No further increase in cell viability was observed after treating the monocytes with additional EC. On the contrary, there was a significant and almost immediate decrease in cellular viability to day 1 values (Auto: 0.259; AU-WF: 0.308; FBS: 0.18; FBS-WF: 0.215 ), and at day 14 very few living cells were found (Figure 1c). In addition, there was no significant difference in cell viability among any of the four media used during the first 7 days of culturing.

\section{Characterization of the cultured cells}

A significant increase in CD68-positive cells from day 1 to 7 in each of the media was observed during cultivation (Figure 2 and Table 2). The highest increase in the ratio of CD68-positive to CD68-negative cells was detected in the media with the growth factors M-CSF and IL-3 (AU-WF, +14.6\%; $P=0.41$ ), with FBS-WF $(+18.3 \%$; $P=0.04)$ being the only medium to generate a significant increase (Figure 2). Media with autologous serum showed an increase in CD68-positive cells as well $(+9.7 \%, P=0.12)$, but the differences were not statistically significant. The media with FBS showed the smallest increase in CD68-positive cells $(+7.1 \%, P=0.07)$. Neither markers of endothelial cells, their progenitors (CD31, CD144, VEGF, VEGF-R2) nor the stem cell marker CD34 were detected in the cultured monocytes (Figure 3a). Staining of the cultured cells with CD14 also showed an increase in CD14-positive cells when grown with media growth factors $(\mathrm{AU}-\mathrm{WF}-\mathrm{MD}=+4.2$; $P=0.457 \quad$ FBS-WF-MD $=+2.3, \quad P=0.332) \quad$ or in Auto $(\mathrm{MD}=+3.1 ; P=0.440)$ from day 1 to 7 (Figure $3 \mathrm{~b}$ ).

\section{The differentiation of monocytes/macrophages to vascular cells}

After 7 days in different culture media followed by cultivation for 7 days in EC, only single cells demonstrated a spindleshape-like phenotype. Morphologically, the cells resembled macrophages with their large cell bodies and round nucleus after the first 7 days (Figure 4). The cobblestone-like structure reported for endothelial cells was not observed. Under the microscope, it could be clearly seen that there were hardly any cells left after 7 additional days in the EC, thus confirming the results of the cell viability assay (Figure 1b). Interestingly, immunohistochemical staining with VEGF and VEGF-R2 did show some positive cells. There were also some cells that stained positive for CD14, CD68, CD13, CD144 and CD106 (data not shown). Their morphology looked peculiar, however, 

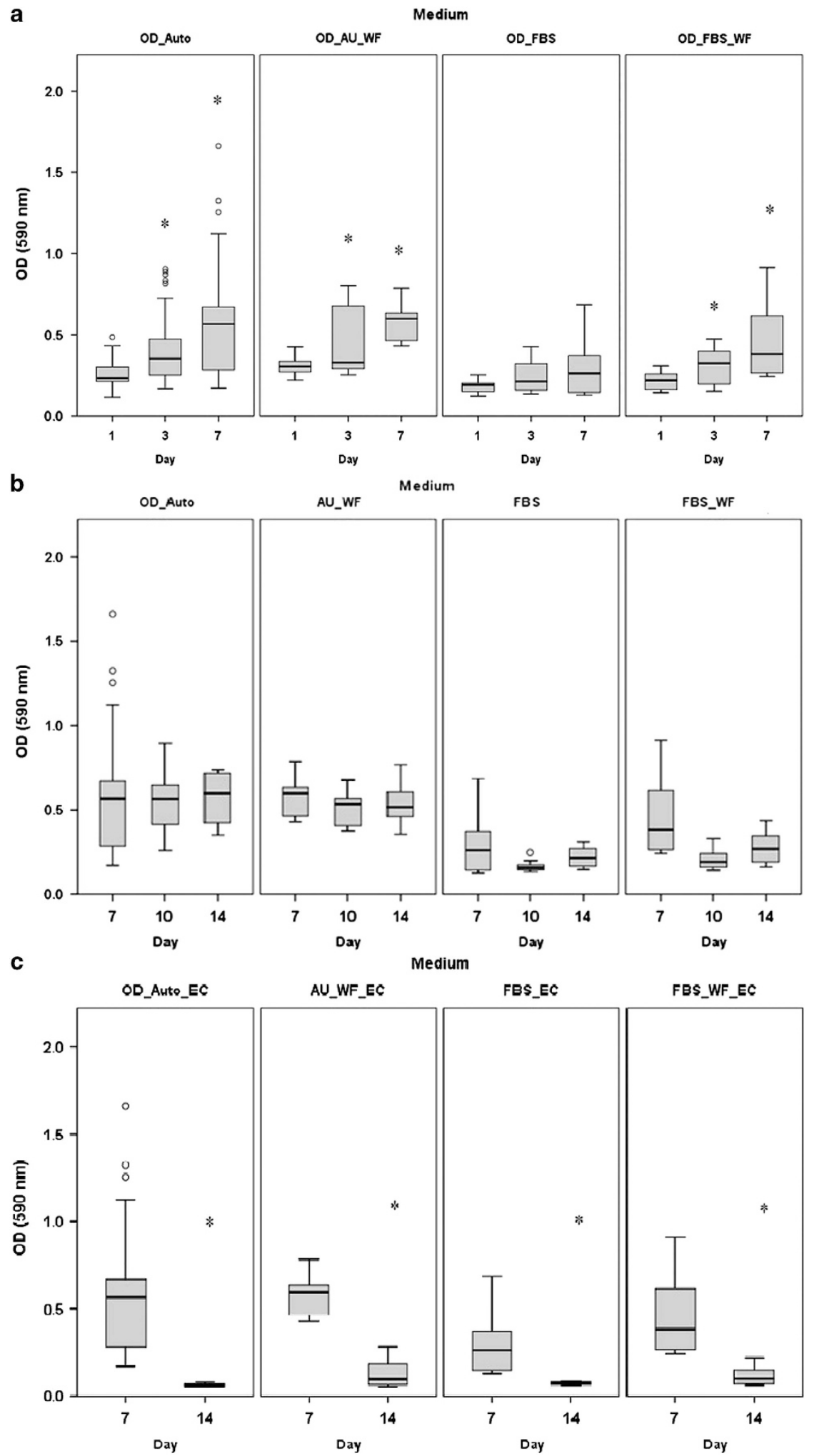

Figure 1 (a) Cell viability from day 1 to 7 in growth media supplemented with either FBS, autologous serum (Auto), autologous serum with M-CSF/IL-3 (Auto+WF) or FBS and M-CSF/IL-3 (FBS+ WF). (b) Cell viability from day 7 to 14 . No significant changes in cell viability could be detected. (c) Cell viability on days 7 and 14 in endothelial cell conditioning medium after previous cultivation in OD_Auto (10\% autologous serum from the corresponding donor), AU-WF (OD_Auto supplemented with $5 \mathrm{ng} \mathrm{ml}^{-1} \mathrm{M}-\mathrm{CSF}$ and $0.4 \mathrm{ng} \mathrm{ml}^{-1} \mathrm{IL}-3$ ), OD_FBS (standard medium with $10 \%$ FBS or OD_FBS_WF (OD_FBS supplemented with $5 \mathrm{ng} \mathrm{ml}^{-1} \mathrm{M}$-CSF and $0.4 \mathrm{ng} \mathrm{ml}^{-1} \mathrm{IL}-3$ ). A significant decrease in cell viability compared with day 1 values can be seen for all cultured cells. Statistically significant increases in cell viability are indicated by an asterisk ('*'). OD, optical density. 


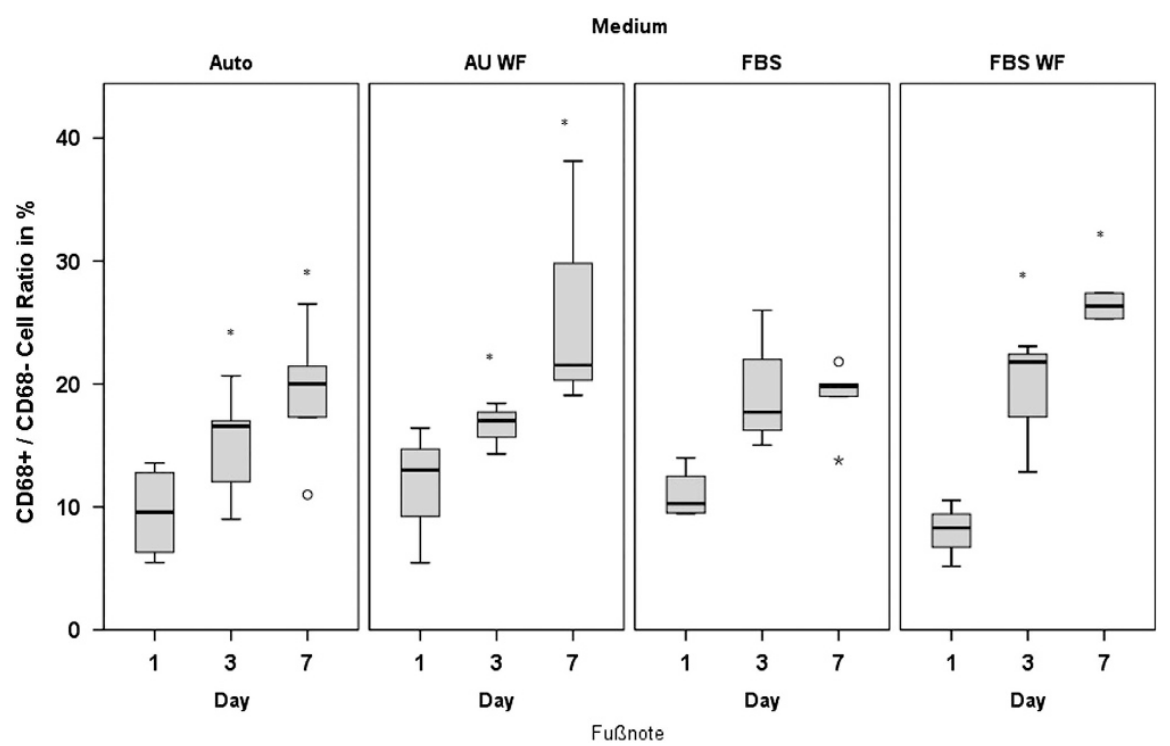

Figure 2 An increase of CD68-positive cells by immunohistochemistry for each of the growth mediums tested was seen. The highest increase was detected for medium supplemented with growth factors. Statistically significant increases in cell viability are indicated by an asterisk (“*’).

Table 2 CD68+/ CD68 - cell ratio for every medium from day 1 to 7

\begin{tabular}{|c|c|c|c|c|c|}
\hline \multirow[b]{2}{*}{ Medium } & \multirow[b]{2}{*}{ Parameter } & \multirow[b]{2}{*}{$B$} & \multirow[b]{2}{*}{ S.e. } & \multicolumn{2}{|c|}{ 95\% confidence interval } \\
\hline & & & & Lower bound & Upper bound \\
\hline \multirow[t]{4}{*}{ AU-WF } & Intercept & 26.250 & 3.992 & 16.483 & 36.017 \\
\hline & $($ day $=1)$ & -14.621 & 5.645 & -28.434 & -0.808 \\
\hline & $($ day $=3$ ) & -9.662 & 5.645 & -23.475 & 4.151 \\
\hline & $($ day $=7)$ & 0 & & & \\
\hline \multirow[t]{4}{*}{ Auto } & Intercept & 19.254 & 2.165 & 14.489 & 24.019 \\
\hline & $($ day $=1)$ & -9.702 & 3.248 & -16.850 & -2.554 \\
\hline & $($ day $=3)$ & -4.196 & 3.062 & -10.936 & 2.543 \\
\hline & $($ day $=7)$ & 0 & $\cdot$ & & \\
\hline \multirow[t]{4}{*}{ FBS } & Intercept & 18.879 & 1.537 & 15.454 & 22.305 \\
\hline & $($ day $=1)$ & -7.872 & 2.306 & -13.011 & -2.734 \\
\hline & $($ day $=3)$ & 0.240 & 2.306 & -4.898 & 5.378 \\
\hline & $($ day $=7)$ & 0 & & & \\
\hline \multirow[t]{4}{*}{ FBS-WF } & Intercept & 26.347 & 2.806 & 19.135 & 33.559 \\
\hline & $($ day $=1)$ & -18.345 & 3.622 & -27.655 & -9.034 \\
\hline & $($ day $=3)$ & -7.116 & 3.622 & -16.427 & 2.195 \\
\hline & $($ day $=7)$ & 0 & . & & \\
\hline
\end{tabular}

and did not resemble the well-known features of endothelial cells. The origin of these cells remains unknown.

\section{Western blot analysis}

GAPDH was detected for every sample (data not shown). CD14 and CD68 were expressed in every sample, with an increase up to seven times the expression at day 1 (Figures $5 \mathrm{a}-\mathrm{c}$ ). The highest increase was detected for cells cultured in media with growth factors. Endothelial markers, such as CD31, CD144, CD106 or VEGEF, could not be detected in any sample on any day. None of the tested antibodies could be detected for cells at day 14 with prior culturing in endothelial cell medium (data not shown).

\section{DISCUSSION}

In the current study, we identified cell culture media additives, such as autologous serum, M-CSF and IL-3, which favor the proliferation of human monocytes in culture and yield significantly higher amounts of cells in comparison to standard medium. The highest cell viability was achieved with all three additives after 7 days of culture. Surprisingly, even if we used commercial EC supplemented with appropriate angiogenic factors, features of endothelial cells could not be detected. Our results were in contrast with previous reports by Kuwana et al. ${ }^{10,13}$ This discrepancy seems to be because these authors differentiated the human monocytes into endothelial cells immediately after their isolation, while we waited 7 days. We suppose that in the isolated CD14+ cells, endothelial progenitors can still exist, which then multiply under suitable cell culture conditions into the desired cell lineage. ${ }^{6}$ Cultivating the monocytes for a longer period of time leads to differentiation into macrophages, as was observed in our study.

The combination of centrifugation and adherence of cells seems to be sufficient to improve the purity of monocytes to $70-80 \%$, as described in earlier studies. ${ }^{18-20}$ The significant increase in the cell viability was observed for up to 7 days. Consequently, this time span appears to be the most suitable for cultivating human primary monocytes. Our main interest was to optimize the cell culture conditions, and particularly to increase the cell numbers. There have been no studies concerning ways to increase cell viability in monocytes. Commonly, 10\% FBS in culture media without any growth 

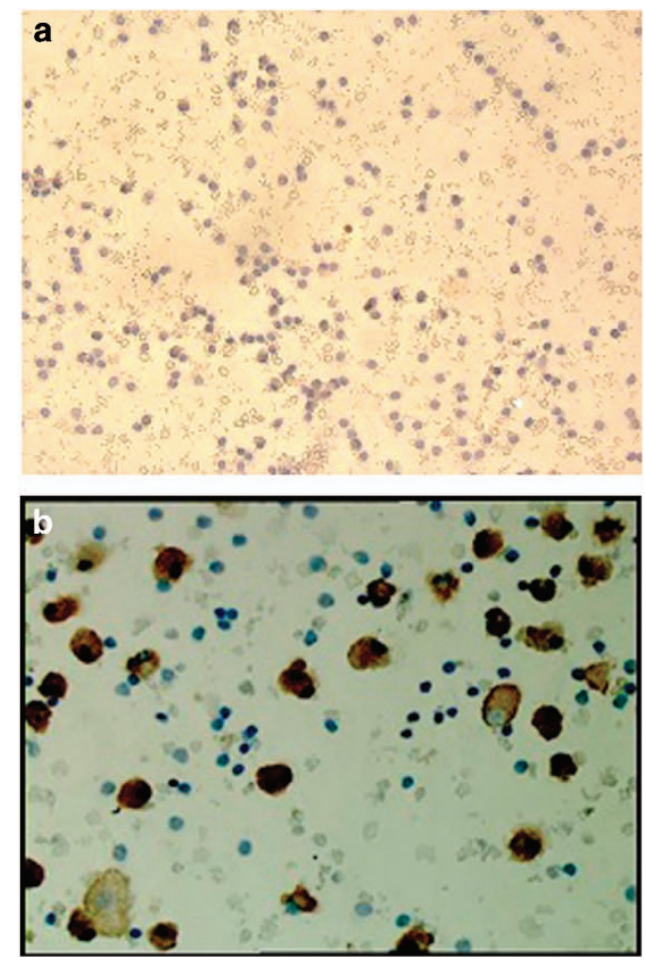

Figure 3 (a) Human cells after isolation of monocytes fixed on a slide with acetone after staining with CD34 antibody. The cells were cultured in medium with 10\% FBS and growth factors at day $7, \times 4$ magnification. CD34 cells are stained brown, negative cells were counterstained with hemalaun (blue). As described in the results, no CD34+ cells were detected. (b) Cultured cells after 7 days in AU-WF medium fixed on a slide with acetone and brown LSAB-HRP staining with CD14-antibody, $\times 8$ magnification. CD14-negative cells are counterstained with hemalaun (blue).

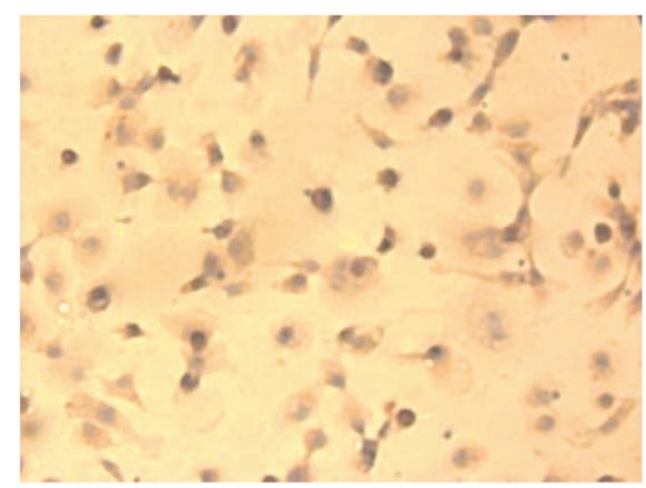

Figure 4 Cells cultured in autologous serum with growth factors on day 7. The small round cells have changed their phenotype to either spindle-shaped cells or cells that are round and have a round nucleus (middle).

factors is used for cultivation of these cells. ${ }^{13}$ In our study, we found that cell culture medium supplemented with autologous serum generated a significantly higher cell yield of cells in comparison to FBS-only. We assume that soluble factors, such as fatty acids, hormones and other unidentified agents, in the serum of the cell donor are responsible for the effect we observed. ${ }^{18}$ In contrast to earlier studies, ${ }^{21}$ we could indeed determine a difference in cell propagation when the donor serum was added to the cell culture medium. Consequently, autologous serum might be considered a promising additive favoring the propagation of human primary monocytes.

Media (either supplemented with autologous serum or FBS) with the growth factors M-CSF and IL-3 showed higher cell propagation for up to 7 days when compared with nonsupplemented cell culture media. Although the effects of these growth factors have already been well-studied, ${ }^{22}$ we were able to show a new aspect of their impact on the propagation of human primary monocytes. They not only positively influence monocyte proliferation, but also their differentiation into macrophages. In contrast, the medium with commercially available FBS alone showed no significant ability to propagate monocytes or to increase their proliferation.

In our study, we were able to show that not only the proper cell culture medium but also time is an important aspect of monocyte propagation. We did not see an improvement in viability for cells cultured longer than 7 days. Consequently, our data recommend the processing of monocytes during this period of time. ${ }^{10,13}$ Based on our observation of the morphology of monocytes during cell propagation, the longer the cells reside in culture, the more of them differentiate into the macrophage-related phenotype. These results were confirmed by immunohistochemical staining and western blot analysis. The presence of growth factors and autologous serum led to an increase in CD68- and CD14-positive cells. ${ }^{23}$ In contrast, medium containing FBS alone showed neither a significant propagation of macrophages nor an impressive upregulation of macrophage markers.

Because autologous serum alone achieved a similar increase in cell viability as medium supplemented with growth factors but a lesser increase in CD68 expression, we assume that the presence of M-CSF and IL-3 accelerated the differentiation of the cells into macrophages more so than favoring their propagation. The upregulation of CD68 and CD14 corresponds with the protein expression pattern of macrophages. Furthermore, the cells also resembled macrophages morphologically. Thus, the cultivation of monocytes in medium supplemented with growth factors increases cell proliferation but leads also to cell differentiation. Our observation was supported by the fact that after 7 days in culture and after subsequent differentiation into endothelial cells, we were not able to detect any endothelial cell markers, such as CD144, CD31, VEGF or VEGF-R2. In contrast to Ruhnke et al..$^{20}$ and others, ${ }^{5,10,13}$ we consider our findings to be a counter-evidence of dedifferentiation of the cultivated monocytes. However, Ruhnke et al. ${ }^{20}$ differentiated monocytes into hepatocytes, not into endothelial cells as was attempted in our study. Furthermore, the authors analyzed various surface markers during the first 6 days of culture and observed an increased expression of CD14 as we did, but almost no expression of CD34, which is also a marker of endothelial cells and therefore supports our findings. Hence, it is possible that monocytes cultivated in 

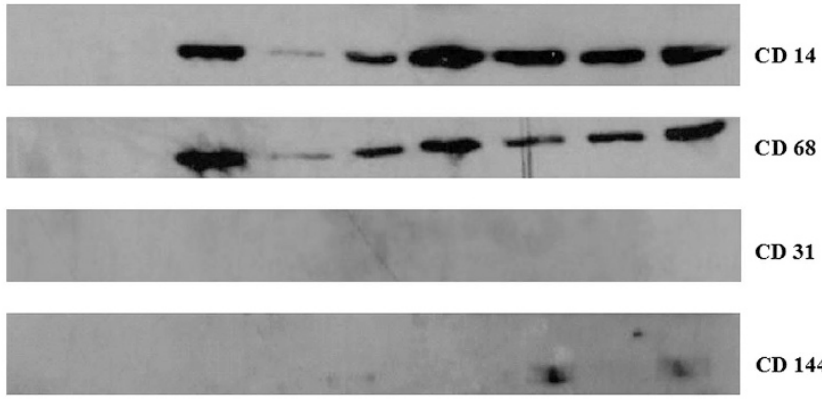

CD 144

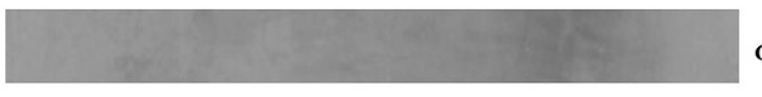

CD 106

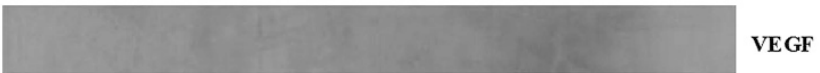

b

Expression CD 68

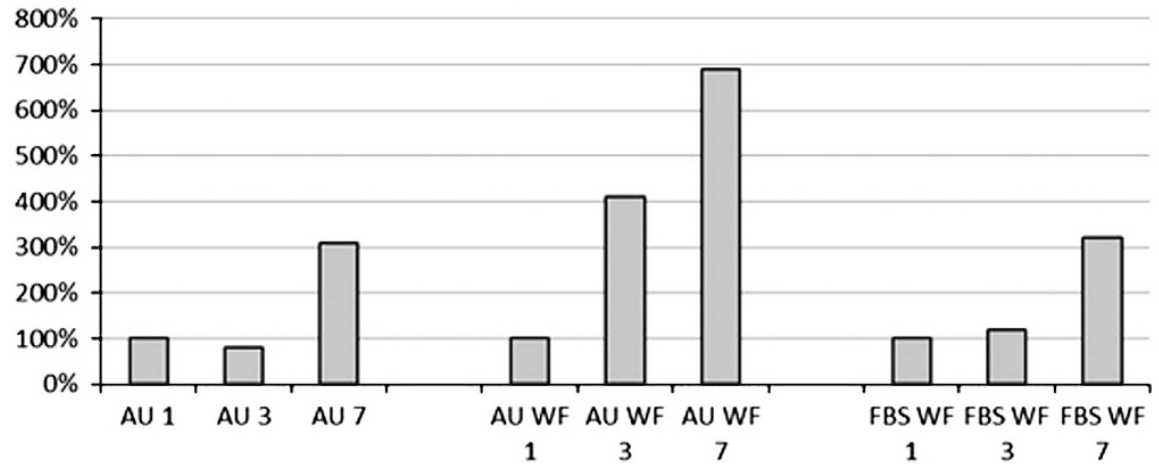

C

Expression CD 14

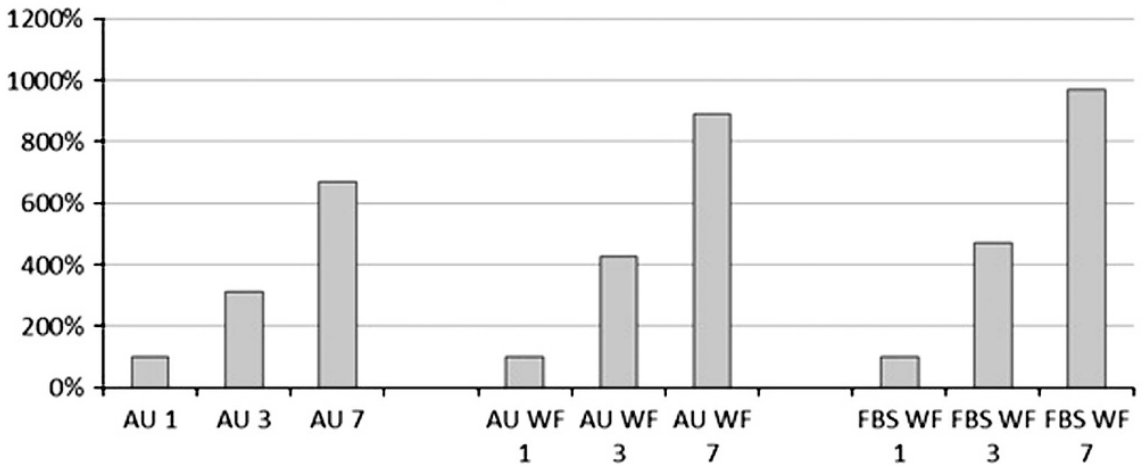

Figure 5 (a) The western blot analysis for media with a significant increase in cell viability, including Auto, AU-WF and FBS-WF. All three media show an increase in protein expression for CD14 and CD68 from day 1 to 7 . The endothelial markers CD144, CD106 and VEGEF were not detected. (b) Protein expression from the western blot analysis showed the greatest increase in CD68 for media supplemented with growth factors after quantification and digitalization. Protein expression of day 1 is set to $100 \%$. (c) Protein expression from western blot analysis shows the greatest increase in CD14 for media supplemented with growth factors after quantification and digitalization. Protein expression of day 1 is set to $100 \%$.

medium containing growth factors are still able to transdifferentiate into hepatocytes but not into the endothelial cell lineage. The ability of monocytes to differentiate into other cells under various growth conditions still has to be further elucidated.

There has been great interest in growing vascular/endothelial cells from monocytes to repair endothelial damage with autologous transplants. During embryogenesis, the commitment of a bipotent stem cell for hematopoetic and endothelial lineages (called a 'hemangioblast') is characterized by the sequential expression of CD144, CD31 and CD34. ${ }^{24,25}$ It has been reported that endothelial progenitors can be selected from the peripheral blood by their expression of CD34, CD133, and VEGFR2 $2^{12,26}$ and that these progenitors also express CD144 
and CD $31 .{ }^{27}$ The differentiation of circulating monocytes into the endothelial lineage is supposed to follow the same sequence of events. ${ }^{10}$ The processing of monocytes in our study failed, however, to differentiate monocytes into endothelial cells. We have to admit that the above mentioned studies used more in-depth analyses by performing flow cytometry and metabolic tests. However, Runhke et al. ${ }^{20}$ Kuwana et al. and others have supported their results using immunohistochemistry as we did. Our immunohistochemical analyses clearly identified the cultured cells as macrophages, and no cobblestone-like morphology characteristic of endothelial cells was observed. One could also argue that differences in the protocols might have caused our failure to yield endothelial cells. Ruhnke et al. ${ }^{20}$ cultured their cells for only $2 \mathrm{~h}$ before scraping the adherent cells, and Kuwana started with a cell population of higher purity by using MACS. However, Ruhnke et al..$^{20}$ also observed macrophages after a prolonged culture period ( $>20$ days), which is in accordance with our results. Furthermore, it seems that the difference in the initial culturing during the 1st day does not lead to different results, as the recommendation is for up to $24 \mathrm{~h}$, but rather, to a different yield of cells. ${ }^{17,19}$ In addition, the differences in the purity of the starting monocytes $(70-80 \%$ vs $>99 \%)$ also do not seem to be crucial for the results. Both Kuwana et al. and Ruhnke et al. ${ }^{10,20}$ succeeded in dedifferentiating their cultured cells.

In summary, the combined protocol of Ruhnke $e^{20}$ and Kuwana for cultivating human primary monocytes failed to differentiate the monocytes into endothelial cells. The procedure used in our study was able to increase the cell viability and proliferation but led mainly to transformation into macrophages, which is the final step in the differentiation process of these hematopoietic stem cells. Thus, we conclude the following: (i) autologous serum and growth factors increase the cell proliferation and cell number for up to 7 days; (ii) all three additives lead to trans-differentiation of monocytes into macrophages. The lowest effect was observed from autologous serum. Hence, autologous serum as an additive seems to be the most suitable for maintaining human primary monocytes in culture; (iii) monocytes are able to differentiate into other cell lineage, however, different protocols are necessary. Based on our results and previous reports, individual protocols are necessary to transform human monocytes into the cell lineage of interest.

\section{CONFLICT OF INTEREST}

The authors declare no conflict of interest.

\section{ACKNOWLEDGEMENTS}

We would like to thank our technician Renate Hegenloh for her expert support during the projects. We would also like to thank Michael Hanna for editing the manuscript as well as Dr Tibor Schuster for supporting the statistical evaluation.
1 Servet-Delprat C, Arnaud S, Jurdic P, Nataf S, Grasset MF, Soulas C et al. Flt3+ macrophage precursors commit sequentially to osteoclasts, dendritic cells and microglia. BMC Immunol 2002; 3: 15.

2 Miyamoto T, Ohneda O, Arai F, Iwamoto K, Okada S, Takagi K et al. Bifurcation of osteoclasts and dendritic cells from common progenitors. Blood 2001; 98: 2544-2554.

3 Kodama H, Inoue T, Watanabe R, Yasuoka H, Kawakami Y, Ogawa S et al. Cardiomyogenic potential of mesenchymal progenitors derived from human circulating CD14+ monocytes. Stem Cells Dev 2005; 14: 676-686.

4 Naito M, Hasegawa G, Takahashi K. Development, differentiation, and maturation of Kupffer cells. Microsc Res Technol 1997; 39: 350-364.

5 Schmeisser A, Garlichs CD, Zhang H, Eskafi S, Graffy C, Ludwig J et al. Monocytes coexpress endothelial and macrophagocytic lineage markers and form cord-like structures in Matrigel under angiogenic conditions. Cardiovasc Res 2001; 49: 671-680.

6 Fernandez Pujol B, Lucibello FC, Gehling UM, Lindemann K, Weidner N, Zuzarte ML et al. Endothelial-like cells derived from human CD14 positive monocytes. Differentiation 2000; 65: 287-300.

7 Harraz M, Jiao C, Hanlon HD, Hartley RS, Schatteman GC. CD34- bloodderived human endothelial cell progenitors. Stem Cells 2001; 19: 304-312.

8 Rehman J, Li J, Orschell CM, March KL. Peripheral blood "endothelial progenitor cells" are derived from monocyte/macrophages and secrete angiogenic growth factors. Circulation 2003; 107: 1164-1169.

9 Urbich C, Heeschen C, Aicher A, Dernbach E, Zeiher AM, Dimmeler S. Relevance of monocytic features for neovascularization capacity of circulating endothelial progenitor cells. Circulation 2003; 108: 2511-2516.

10 Kuwana M, Okazaki Y, Kodama H, Satoh T, Kawakami Y, Ikeda Y. Endothelial differentiation potential of human monocyte-derived multipotential cells. Stem Cells 2006; 24: 2733-2743.

11 Asahara T, Murohara T, Sullivan A, Silver M, van der Zee R, Li T et al. Isolation of putative progenitor endothelial cells for angiogenesis. Science 1997; 275: 964-967.

12 Peichev M, Naiyer AJ, Pereira D, Zhu Z, Lane WJ, Williams M et al. Expression of VEGFR-2 and AC133 by circulating human CD34(+) cells identifies a population of functional endothelial precursors. Blood 2000; 95: 952-958.

13 Kuwana M, Okazaki Y, Kodama H, Izumi K, Yasuoka H, Ogawa Y et al. Human circulating CD14+ monocytes as a source of progenitors that exhibit mesenchymal cell differentiation. J Leukoc Biol 2003; 74 : 833-845.

14 Feldman DL, Mogelesky TC. Use of Histopaque for isolating mononuclear cells from rabbit blood. J Immunol Methods 1987; 102: 243-249.

15 Mosmann T. Rapid colorimetric assay for cellular growth and survival: application to proliferation and cytotoxicity assays. J Immunol Methods 1983; 65: 55-63.

16 Berridge MV, Tan AS. Characterization of the cellular reduction of 3-(4,5-dimethylthiazol-2-yl)-2,5-diphenyltetrazolium bromide (MTT): subcellular localization, substrate dependence, and involvement of mitochondrial electron transport in MTT reduction. Arch Biochem Biophys 1993; 303: 474-482.

17 Dallabrida SM, Ismail N, Oberle JR, Himes BE, Rupnick MA. Angiopoietin1 promotes cardiac and skeletal myocyte survival through integrins. Circ Res 2005; 96: e8-e24.

18 Bennett WE, Cohn ZA. The isolation and selected properties of blood monocytes. J Exp Med 1966; 123: 145-160.

19 Johnson WD Jr, Mei B, Cohn ZA. The separation, long-term cultivation, and maturation of the human monocyte. J Exp Med 1977; 146: 1613-1626.

20 Ruhnke M, Ungefroren H, Nussler A, Martin F, Brulport M, Schormann W et al. Differentiation of in vitro-modified human peripheral blood monocytes into hepatocyte-like and pancreatic islet-like cells. Gastroenterology 2005; 128: 1774-1786.

21 Vogel SN, Perera PY, Hogan MM, Majde JA. Use of serum-free, compositionally defined medium for analysis of macrophage differentiation in vitro. J Leukoc Biol 1988; 44: 136-142.

22 Wang F, Tahara T, Arisawa T, Shibata T, Nakamura M, Fujita H et al. Genetic polymorphisms of CD14 and Toll-like receptor-2 (TLR2) in patients with ulcerative colitis. J Gastroenterol Hepatol 2007; 22: 925-929.

23 Kaluza J. The monoclonal antibody (MAB) CD 68 allows the immunocytochemical identification of macrophages in primary and metastatic brain tumors in paraffin embedded tissues. Folia Histochem Cytobiol 1992; 30: 125-127. 
24 Nishikawa SI, Nishikawa S, Kawamoto H, Yoshida H, Kizumoto M, Kataoka $\mathrm{H}$ et al. In vitro generation of lymphohematopoietic cells from endothelial cells purified from murine embryos. Immunity 1998; 8: 761-769.

25 Yamashita J, Itoh H, Hirashima M, Ogawa M, Nishikawa S, Yurugi T et al. Flk1-positive cells derived from embryonic stem cells serve as vascular progenitors. Nature 2000; 408: 92-96.

26 Gehling UM, Ergun S, Schumacher U, Wagener C, Pantel K, Otte M et al. In vitro differentiation of endothelial cells from $\mathrm{AC} 133$-positive progenitor cells. Blood 2000; 95: 3106-3112.

27 Kuwana M, Okazaki Y, Yasuoka H, Kawakami Y, Ikeda Y. Defective vasculogenesis in systemic sclerosis. Lancet 2004; 364: 603-610. (c) (1) (2) This work is licensed under a Creative Commons Attribution-NonCommercial-ShareAlike $\quad 4.0$

International License. The images or other third party material in this article are included in the article's Creative Commons license, unless indicated otherwise in the credit line; if the material is not included under the Creative Commons license, users will need to obtain permission from the license holder to reproduce the material. To view a copy of this license, visit http://creativecommons.org/licenses/by-nc-sa/4.0/ 